\title{
Benign Metastasizing Leiomyoma of the Lung with Spontaneous Pneumothorax
}

\author{
Ryo Okabe ${ }^{1}$ Tsuyoshi Shoji ${ }^{2}$ Cheng-long Huang ${ }^{2}$ \\ 1 Division of Thoracic Surgery, Matsue Red Cross Hospital, Matsue, \\ Japan \\ 2 Department of Thoracic Surgery, Kitano Hospital, Osaka, Japan \\ Address for correspondence Ryo Okabe, MD, Division of Thoracic \\ Surgery, Matsue Red Cross Hospital, 200 Horo-machi, Matsue, \\ Shimane 690-8506, Japan (e-mail: okabe2006@gmail.com).
}

Thorac Cardiovasc Surg Rep 2013;2:26-28.

\begin{abstract}
Keywords

- benign metastasizing leiomyoma

- pneumothorax

A 47-year-old woman was admitted with recurrent pneumothorax. Preoperative chest computed tomography (CT) showed multiple lung nodules and cysts bilaterally. She had undergone enucleatic myomectomy 12 years earlier. Video-associated thoracoscopic biopsy was performed. Histopathologically, there were bulla-like dilated cystic changes, the walls of which showed spindle cell proliferation, causing pneumothorax. Hormone therapy was started after benign metastasizing leiomyoma resection; pneumothorax has not recurred in 7 months. Multiple residual lung nodules have decreased or disappeared on $\mathrm{CT}$.
\end{abstract}

\section{Introduction}

Benign metastasizing leiomyoma (BML) is a rare benign tumor, occurring commonly in patients with a history of enucleatic or total myomectomy for benign uterine leiomyoma. BML frequently presents with multiple asymptomatic lung lesions, and is usually diagnosed incidentally. A case of BML associated with pneumothorax is reported.

\section{Case Description}

A 47-year-old woman was admitted to our hospital with recurrent pneumothorax in association with the menstrual cycle. Catamenial pneumothorax was suspected, and preoperative chest computed tomography (CT) showed multiple lung nodules and cysts in both lungs (-Figs. 1 and $\mathbf{2}$ ). The patient had undergone enucleatic myomectomy 12 years earlier. 18-Fluorodeoxyglucose positron emission tomography showed no abnormal uptake anywhere in the body. Tumor markers, including carcinoembryonic antigen, squamous cell carcinoma-related antigen, and cytokeratin 19 fragment, were within normal ranges. BML, lymphangioleiomatosis (LAM), and other metastatic tumors were suspected, and video-assisted thoracoscopic biopsy was performed for diagnosis and treatment. A small amount of pleural effusion, dark red lesions in the right middle and lower lobes, and two bullae in the right upper and middle lobes were observed in the thoracic cavity. In addition, one yellow nodule on the right diaphragm and two yellow nodules in the right parietal pleura were observed, but no pores or scattered lesions were observed. In all, four nodules and dark red lesions in the right middle and lower lobes, two bullae in the right upper and middle lobes, and three yellow nodules on the right diaphragm and in the parietal pleura were resected. The nodules in the lung were solid, firm masses with a white cut surface (-Fig. 3). Carbohydrate antigen 125 (CA 125) was increased in the pleural effusion $(1,969 \mathrm{IU} / \mathrm{mL})$. The frozen biopsy results showed spindleshaped smooth muscle cells with mild enlarged nuclei and eosinophilic cytoplasm. There were bulla-like dilated cystic changes, the walls of which showed spindle cell proliferation (-Fig. 4). The tumors were positive for $\alpha$-smooth muscle actin, desmin, and estrogen receptors, and negative for LAM cell marker HMB-45 and CD-10. No uterine endometrial tissue, leiomyoma tissue, or malignant cells was observed in the yellow nodules on the diaphragm and parietal pleura and in the dark red lesions.

Hormone therapy using leuprorelin was started 2 weeks after surgery, and pneumothorax has not recurred in past
License terms Stuttgart · New York

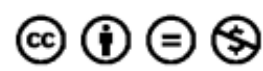

February 17, 2013

accepted after revision

April 9, 2013

published online

April 25, 2013
DOI http://dx.doi.org/ 10.1055/s-0033-1345266. ISSN 2194-7635. 


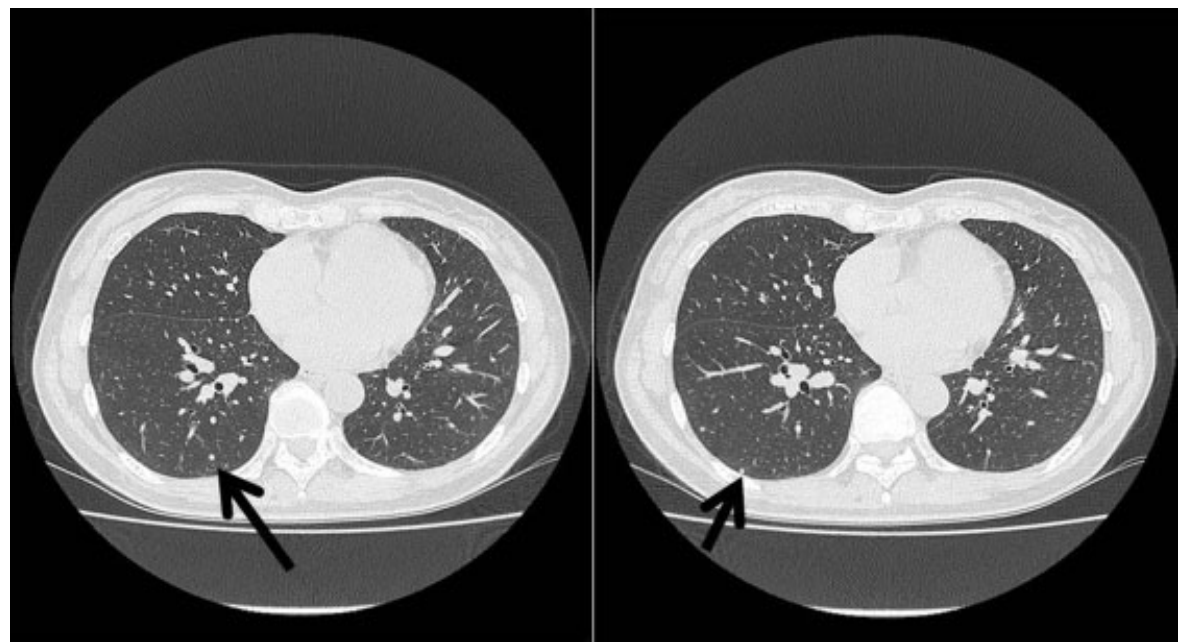

Fig. 1 Chest CT shows multiple, well-circumscribed nodules. The arrow indicates the nodule resected by biopsy.

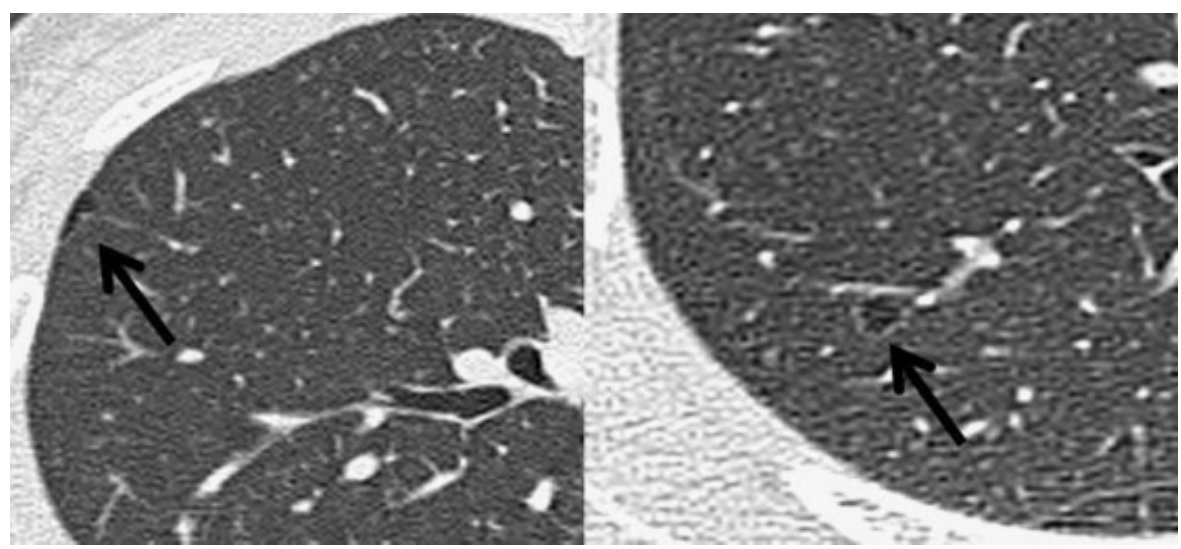

Fig. 2 Preoperative chest CT shows cysts in both lungs (arrows).

7 months. Residual multiple lung nodules have reduced or disappeared on CT.

\section{Discussion}

BML was first described by Steiner in $1939 .^{1}$ This is a rare tumor, with less than 200 cases documented in the literature, and it occurs in the pelvic cavity, lungs, lymph nodes, and, rarely, in the vertebrae or skull base. ${ }^{2}$

BML is suggested by hematogenous lung metastasis of uterine leiomyoma, and there is a history of uterine fibroids in women of reproductive age. Because many cases of postoperative uterine fibroids are enucleated, operation appears to stimulate metastasis. ${ }^{3}$

The prognosis of most cases of BML is good and depends on the degree of estrogen receptor expression. ${ }^{4}$ Some patients die as a result of respiratory insufficiency due to the pulmonary metastases. ${ }^{4}$ Tadashi et al reported a case of BML that decreased in size naturally, suggesting that menopause may play a role in its natural decrease in size. ${ }^{5}$

There is no standard treatment, but the treatment option of choice is primary surgical resection or hormone treatment. After initiation of leuprorelin acetate therapy, total lung capacity increased by $10 \%(500 \mathrm{~mL})$, forced vital capacity increased by $15 \%$ (300 $\mathrm{mL}$ ), forced expiratory volume in the first second increased by $14 \%(270 \mathrm{~mL})$, and diffusion capacity for carbon monoxide increased by $4 \%$ over 11 years of follow-up. ${ }^{3}$

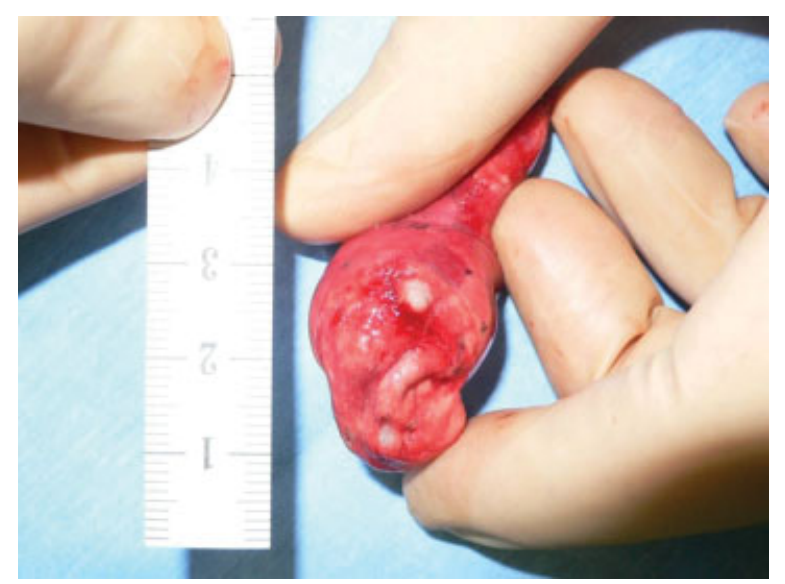

Fig. 3 Pulmonary wedge resection shows well-circumscribed, white nodules. 

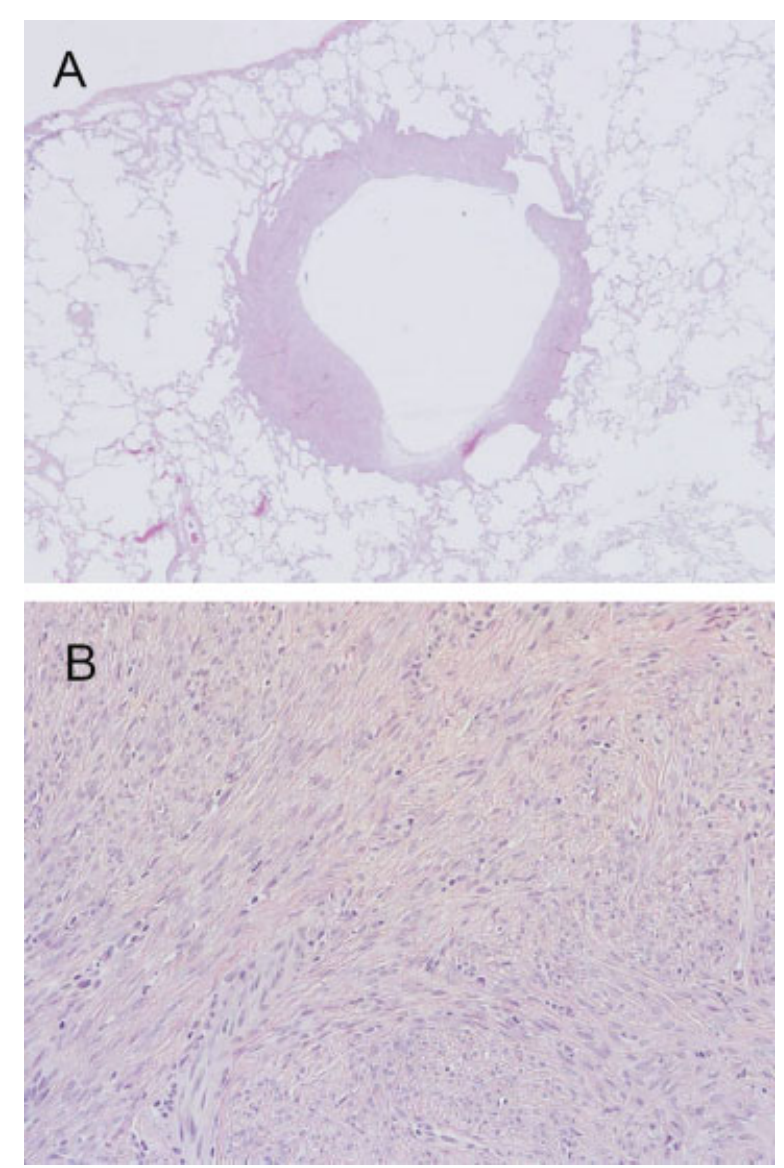

Fig. 4 Frozen biopsy results show: (A) bulla-like dilated cystic changes; (B) the walls of which comprise spindle cells proliferation.

Cystic lesions were observed surrounding the uterine myoma cells in the present patient and, while rare, cases of BML presenting as cystic lesions have been reported, with pneumothorax arising in $1 \%$ of all BML cases. ${ }^{6}$ One proposed mechanism for the development of cystic lesions is gradual growth of the uterine myoma cells in the respiratory tract, which then expands as a result of breakdown of elastic fibers and air trapping, causing cystic lesions to develop. ${ }^{6}$ In the present patient, the mechanism of recurrent pneumothorax suggests that uterine myoma cells in the distal airway became check valves, cystic lesions were formed, and then the cysts ruptured.

Catamenial pneumothorax due to heterotopic endometriosis was initially suspected in the present case; however, pathological examination of the multiple dark red lesions observed intraoperatively in the visceral pleura of the right lung revealed no endometrial tissue. Despite these findings, the repeated occurrence of pneumothorax with menstruation and the elevated CA 125 levels in the pleural effusion fluid prevent coincidence of catamenial pneumothorax due to intrathoracic endometriosis from being completely ruled out. No diagnostic criteria have been established for catamenial pneumothorax. Pores, blueberry spots, and diaphragmatic lesions, such as white, depressed lesions, are observed in $78 \%$ of catamenial pneumothorax cases, and pulmonary pleural lesions are observed in 14 to $39 \%$ of catamenial pneumothorax cases. ${ }^{7}$ The present patient had no diaphragmatic lesions or pelvic endometriosis, and although CA 125 levels in the pleural effusion fluid were inexplicably high, elevated CA 125 levels are also found in benign diseases such as pleurisy, ultimately providing minimal evidence supporting a diagnosis of catamenial pneumothorax.

Regular follow-up is required in the present case due to the possibility of the BML growth.

\section{References}

1 Steiner PE. Metastasizing fibroleiomyoma of the uterus: report of a case and review of the literature. Am J Pathol 1939;15(1): 89-110.7

2 Kang M-W, Kang SK, Yu JH, et al. Benign metastasizing leiomyoma: metastasis to rib and vertebra. Ann Thorac Surg 2011;91(3): 924-926

3 Taveira-DaSilva AM, Alford CE, Levens ED, Kotz HL, Moss J. Favorable response to antigonadal therapy for a benign metastasizing leiomyoma. Obstet Gynecol 2012;119(2 Pt 2):438442

4 Wolff M, Silva F, Kaye G. Pulmonary metastases (with admixed epithelial elements) from smooth muscle neoplasms. Report of nine cases, including three males. Am J Surg Pathol 1979;3(4): 325-342

5 Arai T, Yasuda Y, Takaya T, Shibayama M. Natural decrease of benign metastasizing leiomyoma. Chest 2000;117(3):921922

6 Aboualfa K, Calandriello L, Dusmet M, Ladas G, Hansell DM, Nicholson AG. Benign metastasizing leiomyoma presenting as cystic lung disease: a diagnostic pitfall. Histopathology 2011; 59(4):796-799

7 Alifano M, Jacoblonski C, Kadiri H, et al. Catamenial and noncatamenial, endometriosis-related or nonendmetriosis-related pneumothorax referred for surgery. Am J Respir Crit Care Med 2007;176(10):1048-1053 